\title{
Numerical Investigation on Vortex-Induced Vibration Energy Extraction Efficiency of Double Circular Cylinders In Tandem Arrangement at Low Reynolds Number
}

\author{
Wu Junwu, Yin Zhongjun \\ School of Mechanical Engineering, University of Science and Technology Beijing, 100083, China
}

\begin{abstract}
Vortex shedding from a bluff body results in fluctuating forces acting on the bluff body, which may induce vibration of the bluff body when the bluff body is elastically mounted or deformable. Researchers put forward an idea that we can ex-tract energy from the water flow based on VIV at low flow velocity. Although plenty of researches on parameters of VIV are already presented, however, the improvement of energy extraction efficiency still needs further study. According to the previous research, this essay has simulated flow-induced vibration of tandem double circular cylinders when Reynolds number is 100 . Working condition has been considered as the fixed upstream cylinder and the free vibration of the downstream cylinder. The influence of the mass coefficient and the two cylinders spacing ratio on the downstream cylinder's energy obtained from the fluid is studied. Analysis results show that, the maximum value of the energy extraction efficiency is before the frequency locked range. In the case of large spacing ratio $(\mathrm{L} / \mathrm{D}=7 \sim 9)$, the phenomenon of "beat vibration" appears on the downstream cylinder. The results of this work could provide reference for the improvement of energy extraction efficiency and the design of VIV converter.
\end{abstract}

\section{Introduction}

Vortex shedding from a bluff body results in fluctuating forces acting on the bluff body, which may induce vibrations of the bluff body when the bluff body is elastically mounted or deformable [1]. Vibrations that induced by vortex shedding and wake instability widely exist in nature and engineering field, such as cable bridges, marine risers and ocean platforms [2], and they may destroy the integrity and reliability of structures. However, based on vortex-induced vibration (VIV), renewable energy can be harvested from the flow water at low velocity, which has caused abroad attention in recent years. Bernitsas proposed a single cylinder converter which harvesting energy from water flow based on VIV in the Marine Renewable Energy Laboratory (MRELab) at the University of Michigan [3, 4]. The effects of high damping and high Reynolds number were researched, and the highest efficiency of energy con-version and the corresponding damping value were found (the converter was at a speed between $0.4 \mathrm{~m} / \mathrm{s}$ and $1.1 \mathrm{~m} / \mathrm{s}$ and the spring stiffness between $400 \mathrm{~N} / \mathrm{m}$ and $1800 \mathrm{~N} / \mathrm{m}$ ). A.Barrero-Gil [5] measured the forces acting on the cylinder and the vibration response of single cylinder in crossflow, and the effects of mass ratio and damping ratio on the energy extraction efficiency were dis-cussed. It was found that there is a maximum efficiency when $\mathrm{m}^{*} \xi$ is 0.25 . Moreover, the effects of parameters like $\mathrm{m}^{*}, \xi, \mathrm{m}^{*} \xi$ and nature frequency were studied by $2 \mathrm{D}$ simulation [6]. It was found that the vibration amplitude could increase when double circular cylinders in tandem arrangement ac-cording to experiment and numerical simulation $[7,8]$.

Now there are many researches on the influence parameters of VIV energy extraction of single cylinder based on experimental investigation and numerical simulation. However, it is hard to obtain expression of vibration response of double circular VIV systems because the vibration response is not a single sinusoidal variation and the improvement of energy extraction efficiency still needs further study. In this paper, it focuses on two circular cylinders oscillating at low Reynold numbers by numerical simulation method. The influences of mass ratio and spacing ratio on the energy extraction efficiency were discussed, and the working condition has been considered as the fixed upstream cylinder and the free vibration of the downstream cylinder [9].

\section{Mathematical modeling and numerical simulations}

The numerical simulation of the governing equations of fluid-structure interaction with a freely oscillating cylinder is hard to obtain, because the boundary conditions change as the cylinder moves. The fluid flow is governed by 2-D incompressible Navier-Stokes equations in terms of primitive variables, which can be expressed as follows:

$$
\frac{\partial u}{\partial t}=-(\boldsymbol{u} \bullet \nabla) \boldsymbol{u}-\frac{1}{\rho} \nabla p+\nu \nabla^{2} \boldsymbol{u}+f
$$




$$
\nabla \cdot \boldsymbol{u}=0
$$

Where $\boldsymbol{u}$ is the velocity vector, $t$ is the time, $p$ is the pressure, $\rho$ is density for fluid, $v$ is the kinematic viscosity, $\nabla$ is the gradient operator, and $f$ is the additional volume force vector, which represents the fluid-structure coupled boundary conditions. Laminar model and SIMPLEC methods was adopted.

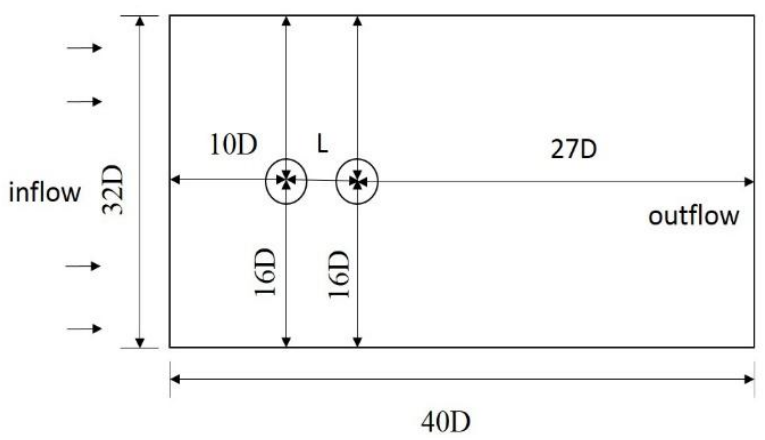

Figure 1. Computational domain in present simulation.

As shown in Figure 1, the sizes of the computational domain are $40 D \times 32 D$, and double circular cylinders are placed in tandem arrangement (the upstream cylinder is fixed and the downstream cylinder single is free in cross flow). Where $\mathrm{D}$ is the diameter of cylinder, $\mathrm{L}$ is the distance between two cylinders.

For the free vibration of the downstream cylinder, the equation of motion for an elastically mounted cylinder of mass $\mathrm{m}$ can be defined as

$$
m \ddot{y}+c \dot{y}+k y=F_{y}(t)
$$

Where $F_{y}$ is provided by the fluid pressure and viscous forces, $y$ is the direction normal to the flow, $\dot{y}$ and $\ddot{y}$ are the velocity and acceleration of the cylinder. To obtain the vibration response of the downstream cylinder, Newmark- $\beta$ was used to solve the Equation 3.

The ratio of harnessed power to the power available in the fluid is

$$
\eta=\frac{P_{\text {harness }}}{P_{\text {fluid }}}=\frac{\frac{1}{2} U_{\infty}^{3}}{\frac{1}{n \Delta t} \sum_{t}^{t+n \Delta t} F_{y} \dot{y} \Delta t}
$$

Where $\eta$ is the energy conversion efficiency, $P_{\text {harmess }}$ is the harnessed power, $P_{\text {fuid }}$ is the total power in fluid, $\Delta t$ is the time step.

\section{Results and discussions}

In this study, the effects of mass ratio and spacing ratio were investigated, and the Reynolds number is 100 . The values of amplitude and the energy conversion of cylinders that placed in tandem arrangement were examined. The working conditions were based on references, in order to verify the numerical simulations results. As it can be seen from Figure 2, the amplitude of the downstream cylinder increases at first and then decreases with increasing Ur (reduced velocity), the trend is in agreement with the references $[7,10]$.

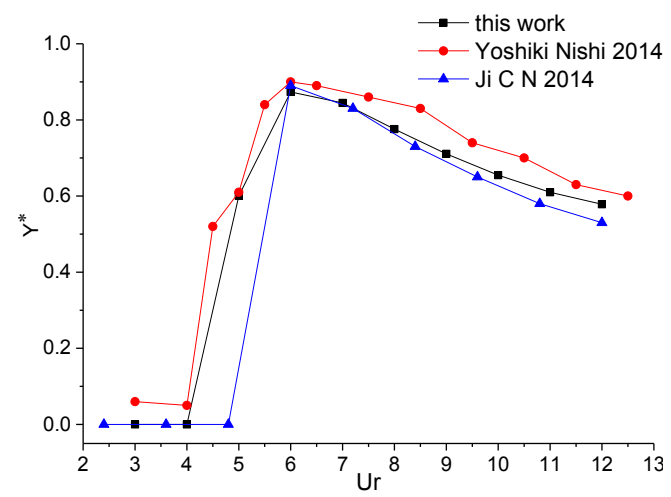

Figure 2. Variation of the amplitude of the downstream cylinder with the reduced velocity When the cylinder spacing ratio is $\mathrm{L} / \mathrm{D}=3$.

\subsection{Mass coefficient}

The conditions are listed in Table 1 , and the vibration response and forces are shown in Figure 3 and Figure 4. The efficiency is related with $F_{y}$ and $y$ : Figure 3 shows the $\mathrm{Y}^{*}$ versus Ur for different $\mathrm{m}^{*}$, when $\mathrm{m}^{*}$ is fixed, the amplitude of the downstream cylinder increases at first and then decreases with increasing Ur. $\mathrm{Y}^{*}$ decreases with the increasing of mass coefficient, when $\mathrm{Ur}$ is fixed. When $\mathrm{Ur}$ is $6 \sim 8, \mathrm{Y}^{*}$ peaks at approximately 0.85 . The vibration was strong in this condition, but Lift coefficient dropped to a low value. The variation of lift coefficient is presented in Figure 4. The Lift coefficient increases to the peak with the increasing of Ur, and then drop fast, finally increases slowly, and the Lift coefficient decreases with the increasing of mass coefficient with fixed Ur. Those cause peak values of the efficiency when Ur are 5 and 8 , and the minimum value when $\mathrm{Ur}=6$.

Table 1. Numerical simulation scheme

\begin{tabular}{|c|c|c|}
\hline Case & $\begin{array}{c}\text { Fixed } \\
\text { parameter }\end{array}$ & $\begin{array}{c}\text { Variable } \\
\text { parameter }\end{array}$ \\
\hline 1 & $\begin{array}{c}\mathrm{L} / \mathrm{D}=3, \\
\zeta=0\end{array}$ & $\begin{array}{c}\mathrm{m}^{*}=1, \mathrm{~m}^{*}=2, \\
\mathrm{~m}^{*}=4\end{array}$ \\
\hline 2 & $\begin{array}{c}\mathrm{m}^{*}=2, \mathrm{Ur}=6, \\
\zeta=0\end{array}$ & $\mathrm{~L} / \mathrm{D}=6 \sim 9$ \\
\hline
\end{tabular}

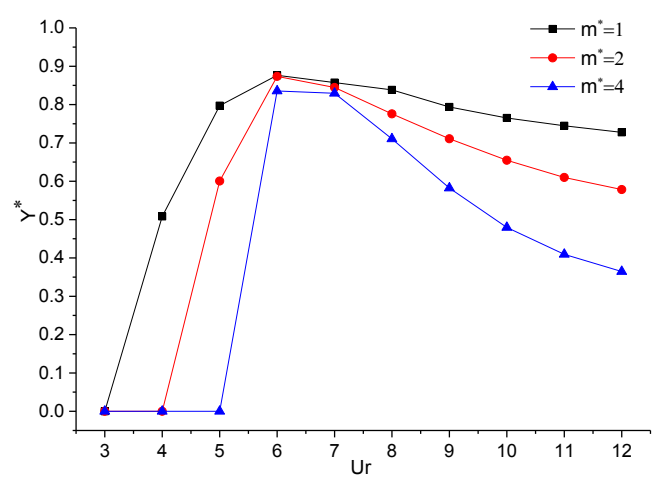

Figure 3. Variation of dimensionless vibration amplitude with the reduced velocity in case 1 


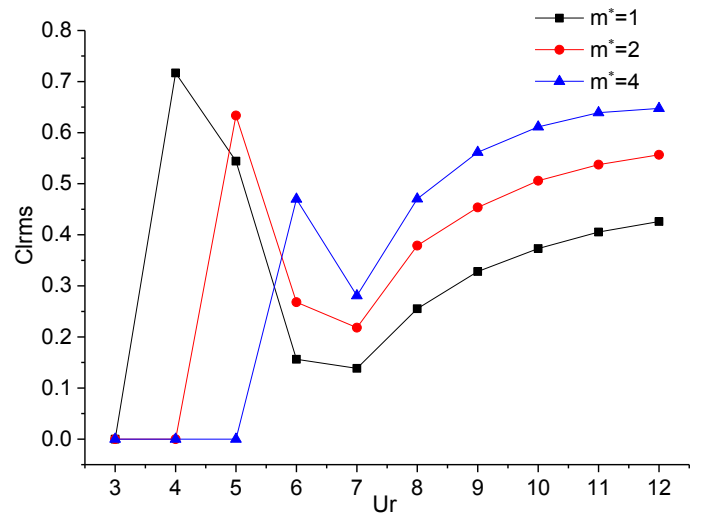

Figure 4. Variation of Lift coefficient root mean square with the reduced velocity in case 1 .

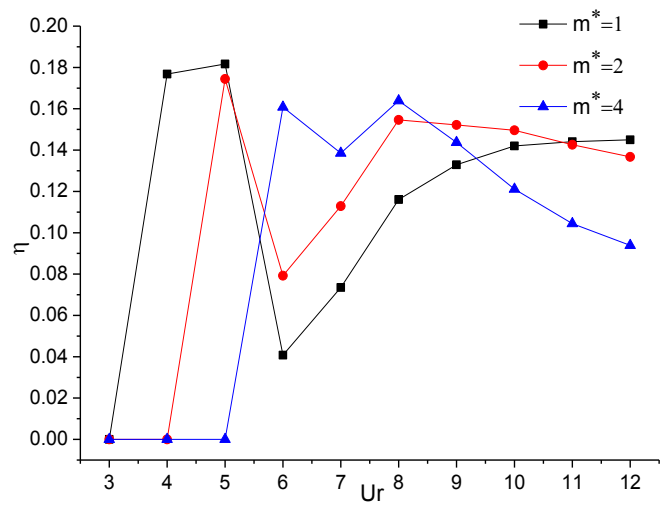

Figure 5. Variation of energy acquisition efficiency with the reduced velocity in case 1 .

\subsection{Space ratio}

The influence of the space ratio is investigated at this part. The conditions are listed in Table 2. Figure 6 shows that the value of efficiency reaches to the maximum when space ratio $\mathrm{L} / \mathrm{D}=4$. When $\mathrm{L} / \mathrm{D}=2 \sim 4$, the $\mathrm{Y}^{*}$ and $\eta$ increase with the increasing of $\mathrm{L} / \mathrm{D}$, and when $\mathrm{L} / \mathrm{D}=4 \sim 6$, though the value of $\mathrm{Y}^{*}$ is still high, the efficiency decreases as a result of the sharply decrease of lift coefficient. While $\mathrm{L} / \mathrm{D}$ is greater than 6 , there is another peak of efficiency, then the efficiency deceases. In all, a higher $\mathrm{Y}^{*}$ can be obtained when the cylinders are placed in tandem arrangement, and the values of the energy extraction efficiency have two maximums when $\mathrm{L} / \mathrm{D}=4$ or 7 .

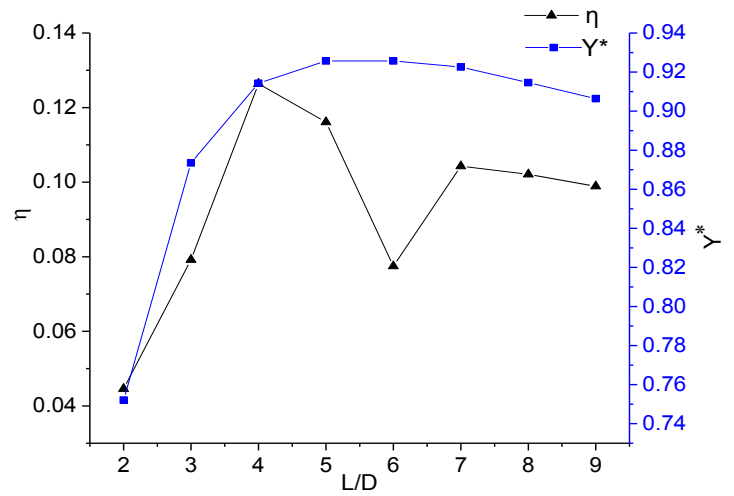

Figure 6. Variation of energy acquisition efficiency and dimensionless vibration amplitude with the space ratio in case 2
In addition, the harnessed power curve of the downstream cylinder when the double circular cylinders that placed in tandem arrangement is not sinusoidal. The phenomenon of "beat vibration" appears on the downstream cylinder when the spacing ratio is large $(\mathrm{L} / \mathrm{D}=7 \sim 9)$.
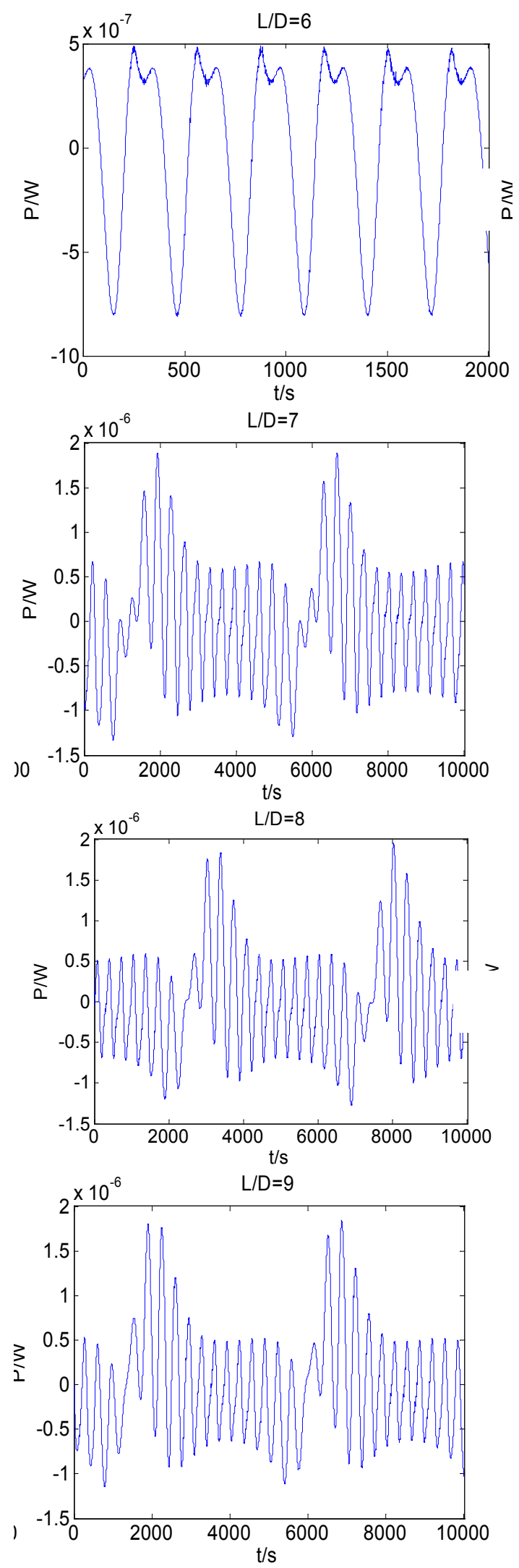

Figure 7. The harnessed power of case 2 . 


\section{Conclusion}

In this work, the influence of the mass coefficient and the spacing ratio of two cylinders on the energy extraction efficiency were discussed, and the following conclusions are obtained:

- The value of the energy extraction efficiency reached maximum when the Ur is 6 or 8 , and the optimum spacing ratio is 4 in this work condition.

- The instantaneous power curve of the downstream cylinder when the double circular cylinders that placed in tandem arrangement is not sinusoidal. The phenomenon of "beat vibration" appears on the downstream cylinder when the spacing ratio is large.

\section{References}

1. P. Bearman, J FLUID STRUCT 27 (2011).

2. Z. Han, D. Zhou, T. He, J. Tu, C. Li, K.C. Kwok, C. Fang, OCEAN ENG 96 (2015).
3. M.M. Bernitsas, K. Raghavan, Y. Ben-Simon, E. Garcia, J OFFSHORE MECH ARCT 130 (2008).

4. J. Lee, M. Bernitsas, OCEAN ENG 38 (2011).

5. A. Barrero-Gil, G. Alonso, A. Sanz-Andres, J SOUND VIB 329 (2010).

6. Z.M. Luo, L.X. Zhang, Journal of Vibration \& Shock 33 (2014).

7. Y. Nishi, Y. Ueno, M. Nishio, L.A.R. Quadrante, K. Kokubun, J SOUND VIB 333(2014).

8. B.S. Carmo, S.J. Sherwin, P.W. Bearman, R.H.J. Willden, J FLUID STRUCT 27 (2011).

9. G.R.S. Assi, J FLUID STRUCT 50 (2014).

10. C. Ji, W. Chen, J. Huang, W. Xu, Chinese Journal of Theoretical \& Applied Mechanics 46 (2014) 